\title{
Frailty predicts worse outcomes after intracranial meningioma surgery irrespective of existing prognostic factors
}

\author{
Brianna C. Theriault, MD, PhD, ${ }^{1}$ Julia Pazniokas, MD, ${ }^{1}$ Anusha S. Adkoli, BS, ${ }^{1}$ Edward K. Cho, BA, \\ Naina Rao, BS, ${ }^{1}$ Meic Schmidt, MD, MBA, ${ }^{3}$ Chad Cole, MD, MSc, ${ }^{2}$ Chirag Gandhi, MD, ${ }^{2}$ \\ William T. Couldwell, MD, PhD, ${ }^{4}$ Fawaz Al-Mufti, MD, ${ }^{2}$ and Christian A. Bowers, MD ${ }^{3}$
}

'School of Medicine, New York Medical College, Valhalla, New York; ${ }^{2}$ Department of Neurosurgery, Westchester Medical Center, Valhalla, New York; ${ }^{3}$ Department of Neurosurgery, University of New Mexico, Albuquerque, New Mexico; and ${ }^{4}$ Department of Neurosurgery, University of Utah, Salt Lake City, Utah

OBJECTIVE Frailty has been recognized as a predictor of adverse surgical outcomes across multiple surgical disciplines, but until now the relationship between frailty and intracranial meningioma surgery has not been studied. The goal of the present study was to determine the relationship between increasing frailty (determined using the modified Frailty Index $[\mathrm{mFI}]$ ) and intracranial meningioma resection outcomes (including hospital length of stay [LOS], discharge location, and reoperation and readmission rates).

METHODS This is a single-center retrospective cohort study of patients who underwent intracranial meningioma resection between August 2012 and May 2018. Seventy-six patients met the inclusion criteria.

RESULTS Frailty was associated with increased hospital LOS $(p=0.0218)$, increased reoperation rate $(p=0.029)$, and discharge to a higher level of care: an inpatient rehabilitation facility or a skilled nursing facility $(p=0.0002)$. After multivariable analysis, frailty was determined to be an independent risk factor for increased LOS, worse discharge disposition, and subsequent readmission.

CONCLUSIONS Frailty is an independent risk factor for worse outcomes following intracranial meningioma resection, including increased LOS, reoperations, and worse discharge disposition. Frailty may help stratify preoperative surgical risk, and thus may provide important clinical information to help neurosurgeons and elderly patients weigh the risks and benefits of resection.

https://thejns.org/doi/abs/10.3171/2020.7.FOCUS20324

KEYWORDS modified Frailty Index; age; meningioma; brain tumor; neurosurgery

$\mathrm{M}$ ENINGIOMAS are the most common primary brain tumor; they are generally benign and slow-growing, and frequently may remain an incidental finding for prolonged periods. ${ }^{1,2}$ Additionally, meningiomas can be associated with significant morbidity and mortality, through their compression of critical neurovascular structures and/or the high associated comorbidity of resection. Resection of symptomatic or enlarging meningiomas is elective; thus, identifying independent risk factors associated with worse outcomes after intracranial meningioma surgery is crucial.

Frailty is broadly defined as a "decrease in physiological reserve," which represents patients' biological age rather than their chronological age, and has recently been used to predict outcomes and complication risk after surgical interventions. ${ }^{3}$ While the medical literature often uses functional frailty measurements, the surgical literature more commonly uses comorbidity-based frailty indices such as the modified Frailty Index (mFI). The mFI was developed by Velanovich et al. by matching factors identified in the Canadian Study of Health and Aging to variables recorded in the National Surgical Quality Improvement Program (NSQIP), which allows for retrospective studies (Table 1). ${ }^{4}$ A large body of neurosurgery literature has recently demonstrated the value of the $\mathrm{mFI}$ in predicting complications among neurosurgical patients. A recent review of the literature on frailty in neurosurgery revealed that the $\mathrm{mFI}$ was used to quantify frailty in 13 of 25 published studies. ${ }^{5}$

ABBREVIATIONS BMI = body mass index; DVT = deep vein thrombosis; $L O S=$ length of stay; $\mathrm{mFI}=$ modified Frailty Index.

SUBMITTED April 18, 2020. ACCEPTED July 17, 2020

INCLUDE WHEN CITING DOI: 10.3171/2020.7.FOCUS20324. 
However, the overwhelming majority of this literature involves spine procedures. ${ }^{6-10}$ In fact, only 3 published studies demonstrated the predictive value of frailty for patients harboring cranial tumors. ${ }^{11-13}$ These studies demonstrated an increase in mortality, ${ }^{11-13}$ complications, ${ }^{11-13}$ length of stay (LOS), ${ }^{12,13}$ and discharge to a higher level of care ${ }^{13}$ in frailer patients following tumor resection.

The goals of the present study were to examine the effect of increasing frailty, that is, poorer functional reserve, on outcomes after meningioma resection.

\section{Methods \\ Study Design and Participant Selection}

This paper describes a retrospective cohort study conducted at Westchester Medical Center in Valhalla, New York; the patients had been treated between August 2012 and May 2018. The study was approved by the center's institutional review board. As this was a retrospective chart review, we received a waiver for patient consent. Eightyeight patients were identified as having "meningioma" according to pathology reports, with 6 patients excluded for having a spinal meningioma and 6 other patients excluded because definitive surgery was not performed at our institution. Therefore, the final cohort sample contained 76 patients.

\section{Measures and Outcomes}

Patient age, sex, body mass index (BMI), smoking status, and tumor size (largest diameter in centimeters) were recorded. The World Health Organization (WHO) tumor grade (per pathology report) and tumor location (per radiology report) were recorded but were not used in the statistical analysis. The incidence of medical complications (deep vein thrombosis [DVT], pulmonary embolism, pneumonia, hyponatremia, and acute kidney injury) as well as surgical complications (new postoperative neurological deficit, postoperative seizures, and wound infections) were recorded. The modified Frailty Index (mFI) was used to measure frailty in this study. Because the required components of this index are routinely included in patient charts, the $\mathrm{mFI}$ is conducive to a retrospective chart review, whereas other frailty measurements requiring specific assessments were not available for this study. The patients' mFIs were determined as previously described by reviewing patient charts and tallying how many mFI variables were identified in each patient (Table 1). ${ }^{4}$ Each $\mathrm{mFI}$ variable is equally weighted and counted as 1 . The patient cohort had an overall low $\mathrm{mFI}(0.86)$, and thus patients were categorized as either non-frail $(\mathrm{mFI}=0)$ or frail $(\mathrm{mFI} \geq 1)$. The primary outcomes were hospital LOS, discharge location, readmission rates, and reoperation rates. We examined frailty's effect on these primary outcomes. Secondary analyses examining the effect of patient age ( $\geq 65$ vs $<65$ years), sex (male vs female), BMI ( $\geq 30$ vs $<30$ ), tumor size $(>3.5$ vs $<3.5 \mathrm{~cm}$ ), or tumor location (skull base vs non-skull base) were conducted on the same outcomes.

\section{Statistics}

All statistical analyses were performed using GraphPad Prism 8.1.2 (GraphPad Software, Inc.) and SPSS (version
TABLE 1. Variables covered in the modified Frailty Index

\begin{aligned} & \hline 1 Functional status 2 (not independent) \\ & \hline 2 History of diabetes mellitus \\ & \hline 3 History of COPD or pneumonia \\ & \hline 4 History of myocardial infarction \\ & \hline 6 History of PCI, PCS, or angina \\ & \hline 7 History of congestive heart failure \\ & \hline 8 History of either peripheral vascular disease or rest pain \\ & \hline 9 History of either transient ischemic attack or CVA \\ & \hline 10 History of CVA w/ neurological deficit \\ & \hline 11 History of impaired sensorium \\ & \hline\end{aligned}

COPD = chronic obstructive pulmonary disease; $C V A=$ cerebrovascular accident; $\mathrm{PCl}=$ percutaneous coronary intervention; $\mathrm{PCS}$ = prior cardiac surgery. Based on information from Velanovich et al.. ${ }^{4}$ each variable is weighted equally (1 point each), and the $\mathrm{mFl}$ is determined by tallying how many variables were identified in each patient. Patients with scores of 1 or greater were designated as "frail." Those with 0 variables were designated as "non-frail."

25, IBM Corp.). For all tests, significance was defined as $\mathrm{p}<0.05$. For the univariate analysis, continuous variables (LOS) were compared between groups using a two-sample $\mathrm{t}$-test. Data are shown as the mean \pm standard error of the mean (SEM). For categorical (binary) variables, groups were compared using Fisher's exact test. Data are shown as odds ratios (ORs) with 95\% confidence intervals (CIs).

Multivariable linear regression was conducted using age, sex, BMI, tumor size, and $\mathrm{mFI}$ as independent variables and LOS as the dependent variable. The results are reported as unstandardized beta coefficients with corresponding $\mathrm{p}$ values. Unstandardized beta coefficients represent the strength of the effect of each individual variable on the dependent variable whereby for every unit change in the independent variable, it is expected that the dependent variable will change by a factor of the beta coefficient.

Multivariable logistic regression was used to predict the effect of the independent variables listed above on categorical dependent variables (e.g., discharge location, readmission, and reoperation rates). Results are represented as ORs with corresponding $\mathrm{p}$ values.

For all statistical tests, significance is defined as $\mathrm{p}<$ 0.05 .

\section{Results}

\section{Demographics and Descriptive Statistics}

Seventy-six patients, who had undergone craniotomy for resection of an intracranial meningioma between $\mathrm{Au}-$ gust 2012 and May 2018, met the study's inclusion criteria (Fig. 1). Table 2 displays patient characteristics for the overall sample as well as for each frailty group separately.

The cohort was $72.6 \%$ female, with a mean age of 55.8 \pm 1.8 years and a mean BMI of $27.9 \pm 0.64$. The mean $\mathrm{mFI}$ was 0.86 . Thirty-four patients had no $\mathrm{mFI}$ variables and were therefore given a score of $\mathrm{mFI}=0$ and categorized as "non-frail." Forty-two patients had one or more mFI variables and were categorized as "frail." Frail patients 


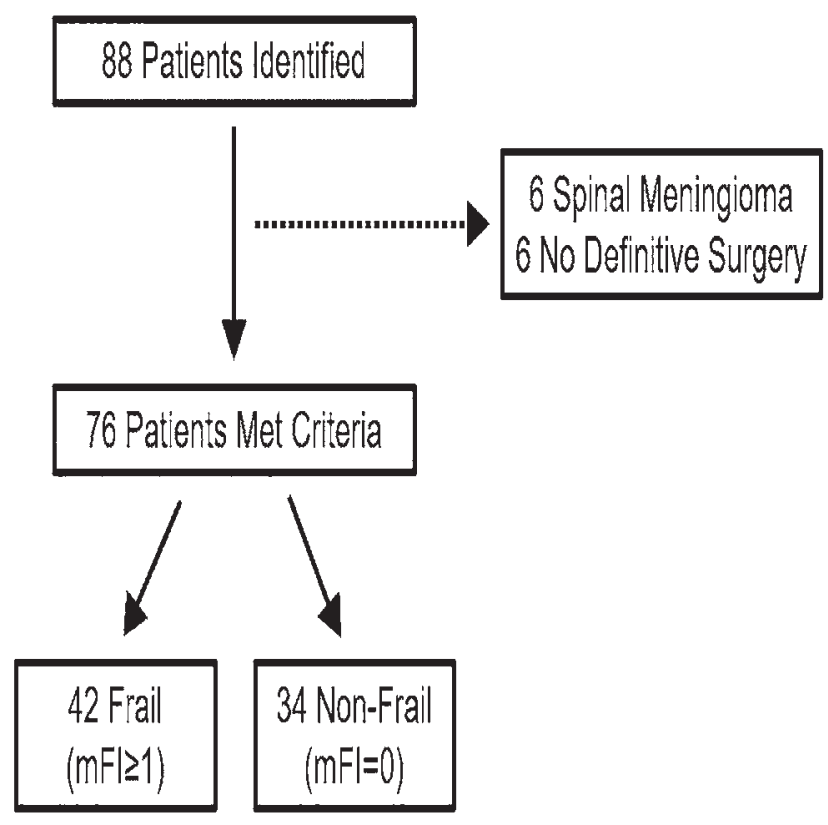

FIG. 1. Flow diagram showing patient selection.

were more likely to be older (mean age $64.1 \pm 2$ vs $45.6 \pm$ 1.92 years, $\mathrm{p}<0.0001$ ) and more likely to have a greater BMI (29.0 \pm 0.86 vs $26.5 \pm 0.91, \mathrm{p}=0.046)$. There was no difference in sex between the two groups $(p=0.31)$ (Table 2). The most common mFI variables were a history of hypertension requiring medications $(31.5 \%)$ and a history of diabetes (23\%) (Table 3). Only 5.3\% of patients had mFI scores greater than 2 (Table 4).

Tumor characteristics between the groups are displayed in Table 2. Frail patients had larger (4.63 vs $3.67 \mathrm{~cm}$ at the largest diameter, $p=0.009)$ and lower-grade $(p=0.0015)$ tumors than non-frail patients. However, although not statistically different, frail patients proportionally had fewer tumors located in a skull base location, while non-frail patients overwhelmingly had skull base tumors $(\mathrm{p}=0.2439)$ (Table 2).

There were no surgical complications in either group. Only one medical complication (DVT) occurred in a single patient in the frail group (Table 2).

\section{Postoperative Outcomes-Univariate Analysis}

Frail patients $(\mathrm{mFI} \geq 1)$ had increased LOSs $(\mathrm{p}=$ 0.0218) (Fig. 2). The LOS trended upwards with increasing mFI scores. Additionally, frail patients were 6 times more likely to be discharged to a higher level of care (skilled nursing facility or inpatient rehabilitation) (OR $6.713,95 \%$ CI 2.498-19.27, p = 0.0002) (Table 5). Frail patients were also more likely to require reoperation (OR $\infty$, $95 \%$ CI $1.205-\infty, p=0.029$ ). Readmission rates between frail and non-frail patients were not significantly different (OR 5.161, CI 1.196-24.61, $\mathrm{p}=0.0545)$. Critically, older age alone ( $\geq 65$ years) did not increase patients' LOS ( $p$ $=0.2672)$, readmission $(\mathrm{p}=0.3182)$ or reoperation $(\mathrm{p}=$ 0.3505 ) rate, or discharge to an inpatient rehabilitation or a skilled nursing facility $(\mathrm{p}=0.0754)$ (Table 5).
TABLE 2. Cohort demographics and tumor characteristics

\begin{tabular}{lcccc}
\hline & $\begin{array}{c}\text { All Patients } \\
(\mathrm{n}=76)\end{array}$ & $\begin{array}{c}\text { Non-Frail } \\
(\mathrm{n}=34)\end{array}$ & $\begin{array}{c}\text { Frail } \\
(\mathrm{n}=42)\end{array}$ & $\mathrm{p}$ Value \\
\hline Mean age (SD), yrs & $55.8(15.3)$ & $45.6(11.2)$ & $64.1(13.1)$ & $<0.0001$ \\
\hline Sex & & & & 0.31 \\
\hline Male & $23(30.2 \%)$ & $8(23.5 \%)$ & $15(35.7 \%)$ & \\
\hline Female & $53(72.6 \%)$ & $26(76.5 \%)$ & $27(64.3 \%)$ & \\
\hline Mean mFI & 0.86 & 0 & 1.57 & \\
\hline Mean BMI & 27.9 & 26.5 & 29 & 0.046 \\
\hline Tumor size, cm & 4.15 & 3.67 & 4.63 & 0.009 \\
\hline Tumor grade & \multicolumn{5}{c}{0.0015} \\
\hline Grade I & $39(51.3 \%)$ & $11(32.3 \%)$ & $28(66.6 \%)$ & \\
\hline Grade II & $35(46.1 \%)$ & $23(67.6 \%)$ & $12(28.6 \%)$ & \\
\hline Grade III & $2(2.6 \%)$ & $0(0 \%)$ & $2(4.8 \%)$ & \\
\hline Tumor location & & & & 0.2439 \\
\hline Skull base & $48(63.2 \%)$ & $24(70.6 \%)$ & $24(57.1 \%)$ & \\
\hline Non-skull base & $28(36.8 \%)$ & $10(29.4 \%)$ & $18(42.9 \%)$ & \\
\hline Complications* & & & & \\
\hline Medical & $1(1.3 \%)$ & 0 & 0 & 0 \\
\hline Surgical & 0 & 0 & \\
\hline
\end{tabular}

Patients designated as non-frail had an $\mathrm{mFI}$ equivalent to 0 ; patients designated as frail had an $\mathrm{mFI} \geq 1$. Values are presented as the number of patients (\%) unless otherwise indicated. Boldface type indicates statistical significance. * Medical complications include DVT, pulmonary embolism, pneumonia, hyponatremia, and acute kidney injury. Surgical complications include a new postoperative neurological deficit, postoperative seizures, and wound infection.

\section{Multivariable Linear and Logistic Regression}

Multivariable linear regression was used to examine the effect of independent variables (e.g., age, BMI, sex, tumor size, $\mathrm{mFI}$ ) on the continuous dependent variable (e.g., LOS) (Table 6). Assumptions for conducting multivariable linear regression analyses were met, including multivariate normality, absence of outliers, and absence of multicollinearity. Increased $\mathrm{mFI}$ was the only independent risk factor associated with an increased LOS that was independent of age, BMI, sex, and tumor size. For every unit increase in the $\mathrm{mFI}$, the expected LOS increased by 1.678 days on average, holding other variables constant $(\mathrm{p}$ $=0.046$ ). On multivariate logistic regression, an increased $\mathrm{mFI}$ was also associated with a significantly increased likelihood of discharge to a higher level of care $(p=0.001)$ and to a higher readmission rate $(\mathrm{p}=0.025)$ (Table 6).

\section{Discussion}

This is the first reported study of the effect of frailty on intracranial meningioma surgery. Frailty was found to be an independent risk factor for increased LOS, discharge to a higher level of care, and readmission rate for patients of all ages. On the univariate analysis, frail patients were also found to be more likely to require reoperation. Importantly, increased age ( $\geq 65$ years) alone was not an independent risk factor associated with worse outcomes.

As an independent risk factor for worse outcomes following intracranial meningioma surgery, frailty has tremendous potential for risk stratification and outcome pre- 
TABLE 3. Distribution of $\mathrm{mFI}$ variables in sample

\begin{tabular}{lcc}
\hline \multicolumn{1}{c}{ mFI Variable } & No. of Patients & $\%$ \\
\hline History of HTN requiring medication & 24 & 31.5 \\
\hline History of diabetes & 20 & 23 \\
\hline Functional status 2 & 13 & 15 \\
\hline History of impaired sensorium & 8 & 9.3 \\
\hline History of COPD or pneumonia & 6 & 7 \\
\hline History of PCl, PCS, or angina & 4 & 4.7 \\
\hline History of stroke w/ deficits & 2 & 2.3 \\
\hline History of CHF & 1 & 1.2 \\
\hline History of MI & 1 & 1.2 \\
\hline History of PVD & 1 & 1.2 \\
\hline History of TIA or stroke & 0 & 0 \\
\hline
\end{tabular}

$\mathrm{CHF}=$ congestive heart failure; $\mathrm{HTN}=$ hypertension; $\mathrm{MI}=$ myocardial infarc tion; PVD = peripheral vascular disease; TIA = transient ischemic attack

diction. These surgeries are almost exclusively elective. Although frail patients were more likely to have larger tumors, frailty remained predictive of outcomes after the multivariate analysis, even after multivariate regression was performed to account for increased tumor size and patient age.

The difference in tumor size in our sample can be explained by the fact that as patients age and become frailer, they also become worse surgical candidates. A surgical selection bias, commonplace in retrospective chart reviews, is demonstrated in this cohort. Older frail patients with small tumors tend to be observed with serial imaging, due to the higher risk of surgical intervention in this age group. Younger, less frail patients with small tumors tend to be offered surgical intervention, given the lower risk of operative intervention in that age group. For this reason, there is a significant difference in tumor size between the frail and non-frail groups: as older and more frail patients harbor meningiomas approaching the size of $5 \mathrm{~cm}(4.63 \mathrm{~cm})$, surgeons and patients believe that surgery becomes less optional and more indicated, because of the neurological symptoms that frequently accompany $5-\mathrm{cm}$ meningiomas.

At first consideration, the $50 \%$ grade II meningioma rate is shockingly high, since it is a 10 -fold increase from the normal average 5\% rate. However, our institution's neuropathological practice is adamant about labeling the hint of possible brain invasion as a grade II. Most of these "grade

TABLE 4. Modified Frailty Index value distribution

\begin{tabular}{ccc}
\hline & \multicolumn{2}{c}{ No. of Patients (\%) } \\
\cline { 2 - 3 } & Frequency & Cumulative Frequency \\
\hline 0 & $34(44.7)$ & $34(44.7)$ \\
\hline 1 & $26(34.2)$ & $60(78.9)$ \\
\hline 2 & $12(15.7)$ & $72(94.6)$ \\
\hline 3 & $2(2.6)$ & $74(97.3)$ \\
\hline 4 & $1(1.3)$ & $75(98.6)$ \\
\hline 5 & $0(0)$ & $75(98.6)$ \\
\hline 6 & $1(1.3)$ & $76(100)$ \\
\hline
\end{tabular}
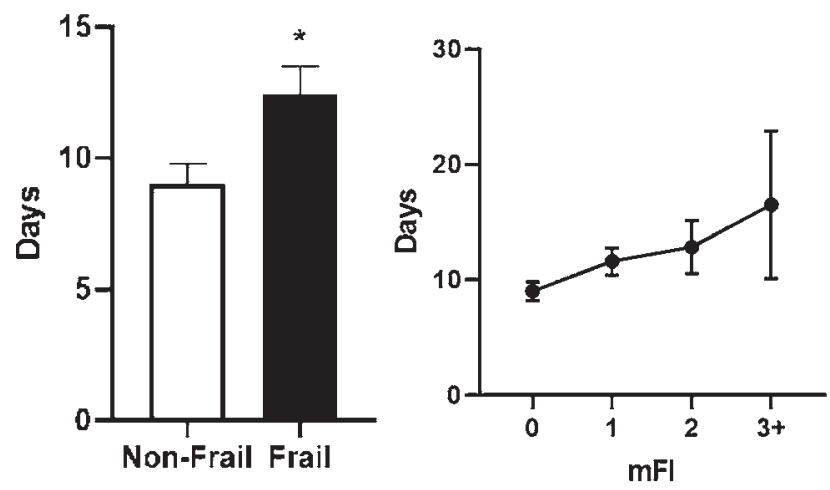

FIG. 2. A bar graph demonstrating LOS in the two patient groups (left) and a line graph showing LOS stratified by patients' mFI (right).

II" meningiomas act like typical grade I tumors, so we tend to observe them with serial imaging after resection.

Tumor location (skull base vs non-skull base) did not confound these results, since the proportional percentage of non-skull base tumors was higher in frail patients. Typically, a meningioma located more peripherally or along the convexities is more surgically accessible and would, therefore, be associated with lower postoperative morbidity. Therefore, these results strengthen the evidence of frailty's influence, since frail patients showed worse outcomes despite the more ideal location of their tumors.

The lower overall mean $\mathrm{mFI}$ in this patient cohort was almost 1 (0.86) and, although the neurosurgery literature on frailty has a variety of methods of frailty definition and stratification for analysis, one key objective of this paper was to closely examine how healthy older patients fare after meningioma surgery. This helped us stratify frailty into frail $(\mathrm{mFI} \geq 1)$ and non-frail $(\mathrm{mFI}<1)$. Therefore, our study's conclusion that non-frail patients of all ages obtain better outcomes after meningioma resection can be reframed in the context of "robustness," or a distinct lack of frailty. Our results indicate that patients who are robust $(\mathrm{mFI}=0)$ have a high physiological reserve and are able to tolerate meningioma resection better than their non-robust, or frail, peers. This conclusion may be particularly important not only for the elderly, but also for young patients diagnosed with meningioma. For elderly patients, robustness may merit consideration of surgical intervention in a patient who traditionally may not otherwise have been considered a candidate for surgery based on advanced age alone.

Additionally, the benign natural history of meningioma is the reason that identification of patients at particularly high or low risk for a poor postoperative course is critical. Increasing use of imaging modalities results in higher rates of incidentally discovered meningiomas. ${ }^{2}$ The slowor nongrowing nature of these tumors makes the majority of surgeries elective in nature or at least nonurgent. This allows for the possibility of preoperative interventions, or "pre-hab" to optimize surgical readiness. ${ }^{14}$ Although there are no studies demonstrating a benefit of prehabilitation for neurosurgical patients, it is possible that presurgical interventions aimed at decreasing frailty could improve outcomes, and this area merits further investigation. 
TABLE 5. Results of the univariate analyses

\begin{tabular}{|c|c|c|c|c|c|}
\hline Dependent Variable & Independent Variable & T Statistic $\left(\mathrm{T}_{\text {crit }} 1.922\right)$ & p Value & Univariate OR $(95 \% \mathrm{Cl})$ & $\mathrm{p}$ Value \\
\hline \multirow{5}{*}{ LOS } & $\mathrm{mFI}$ & 2.344 & 0.0218 & & \\
\hline & Age & -1.118 & 0.2672 & & \\
\hline & BMI & 0.68 & 0.4967 & & \\
\hline & Tumor size & -0.428 & 0.1573 & & \\
\hline & Sex & 0.0276 & 0.978 & & \\
\hline \multirow{5}{*}{ Discharge location } & $\mathrm{mFI}$ & & & $6.713(2.498-19.27)$ & 0.0002 \\
\hline & Age & & & $2.795(0.9634-7.286)$ & 0.0754 \\
\hline & BMI & & & $1.719(0.6795-4.418)$ & 0.3378 \\
\hline & Tumor size & & & $1.987(0.7992-5.093)$ & 0.2324 \\
\hline & Sex & & & $0.6868(0.2722-1.874)$ & 0.6181 \\
\hline \multirow{5}{*}{ Readmission rate } & $\mathrm{mFI}$ & & & 5.161 (1.196-24.61) & 0.0545 \\
\hline & Age & & & $1.933(0.5802-6.907)$ & 0.3182 \\
\hline & BMI & & & $0.7407(0.2305-2.488)$ & 0.7557 \\
\hline & Tumor size & & & $0.7854(0.2285-2.531)$ & 0.7509 \\
\hline & Sex & & & $0.5727(0.1580-2.356)$ & 0.5318 \\
\hline \multirow{5}{*}{ Reoperation rate } & $\mathrm{mFI}$ & & & $\infty(1.205-\infty)$ & 0.029 \\
\hline & Age & & & $2.632(0.5668-11.88)$ & 0.3505 \\
\hline & BMI & & & $1.2(0.2020-6.181)$ & 0.9999 \\
\hline & Tumor size & & & $0.556(0.1235-2.540)$ & 0.6636 \\
\hline & Sex & & & $0.4364(0.3559-3.657)$ & 0.6611 \\
\hline
\end{tabular}

For continuous dependent variables, a two-sample t-test was used and T statistics are reported. For categorical dependent variables, Fisher's exact test was used and ORs are reported. Boldface type indicates statistical significance.

TABLE 6. Results of the multivariate analyses

\begin{tabular}{|c|c|c|c|c|c|}
\hline Dependent Variable & Independent Variable & Unstandardized $\beta$ Coefficient & p Value & Multivariate OR $(95 \% \mathrm{Cl})$ & $\mathrm{p}$ Value \\
\hline \multirow{5}{*}{ LOS } & $\mathrm{mFI}$ & $1.678(0.034-3.322)$ & 0.046 & & \\
\hline & Age & $-0.029(-0.141$ to 0.083$)$ & 0.606 & & \\
\hline & BMI & $-0.201(-0.467$ to 0.066$)$ & 0.138 & & \\
\hline & Tumor size & $0.769(-0.185$ to 1.724$)$ & 0.112 & & \\
\hline & Sex & $-0.493(-2.785$ to 3.772$)$ & 0.765 & & \\
\hline \multirow{5}{*}{ Discharge location } & $\mathrm{mFI}$ & & & $0.077(0.018-0.331)$ & 0.001 \\
\hline & Age & & & $1.465(0.347-6.179)$ & 0.603 \\
\hline & BMI & & & $3.196(0.885-11.538)$ & 0.076 \\
\hline & Tumor size & & & $0.540(0.173-1.689)$ & 0.29 \\
\hline & Sex & & & $0.449(0.131-1.542)$ & 0.203 \\
\hline \multirow{5}{*}{ Readmission rate } & $\mathrm{mFI}$ & & & $0.131(0.022-0.773)$ & 0.025 \\
\hline & Age & & & $1.300(0.311-5.445)$ & 0.719 \\
\hline & BMI & & & $1.174(0.299-4.610)$ & 0.819 \\
\hline & Tumor size & & & $1.379(0.364-5.228)$ & 0.636 \\
\hline & Sex & & & $0.552(0.123-2.474)$ & 0.437 \\
\hline \multirow{5}{*}{ Reoperation rate } & $\mathrm{mFI}$ & & & $0(0-\infty)$ & 0.998 \\
\hline & Age & & & $1.052(0.177-6.239)$ & 0.956 \\
\hline & BMI & & & $0.360(0.055-2.364)$ & 0.287 \\
\hline & Tumor size & & & $2.238(0.378-13.266)$ & 0.375 \\
\hline & Sex & & & $0.194(0.018-2.041)$ & 0.172 \\
\hline
\end{tabular}

For continuous dependent variables (LOS), a multivariable linear regression was carried out and unstandardized beta coefficients are reported. Results are to be interpreted as follows: for every 1 unit increase in the independent variable, the dependent variable changed by a factor of the unstandardized beta coefficient (e.g., a 1-unit increase in the $\mathrm{mFI}$ correlated with a 1.678-fold increase in LOS) holding all other variables constant. For categorical dependent variables, a multivariable logistic regression was carried out and ORs are reported. Boldface type indicates statistical significance. 
This study adds to the body of literature on frailty in neurosurgery by demonstrating that frailty is predictive of outcomes following elective surgery for a benign entity. No previous studies have demonstrated the effect of frailty specific to meningioma resection, despite the fact that meningiomas are the most common primary brain tumor.

\section{Limitations}

There are two primary limitations to this study: its retrospective design and its small sample size.

Any retrospective chart review is subject to several limitations. Importantly, retrospective designs can only establish association rather than causation. Additionally, because the data were not collected in a controlled, prospective manner, it is possible that study variables may have been recorded incorrectly or even be absent from the record. Fortunately, in the present study, there were no missing data. This is likely due to the fact that $\mathrm{mFI}$ variables are simply elements of a patient history that are found documented in several areas of the patient chart.

In many tumor outcomes studies, authors prospectively examine outcomes related to medical or surgical complications, neurological or functional status, and wound complications. Measures for these variables were collected in the chart, but fortunately, except for one case of DVT, we did not observe such complications in our cohort. Further studies examining these variables prospectively are warranted and would bolster the frailty and outcomes literature.

An additional limitation is our small sample size. Studying a benign disease that has a very slow growth process is challenging, because very frail patients with very mild symptoms or incidental lesions most likely would not have undergone surgery, thus lowering the overall mean mFI in this series when compared to other cohorts. Furthermore, non-frail patients with very mild symptoms may have been offered surgery, and some of these patients may have elected resection over observation. Therefore, while we provide statistically significant evidence that the mFI can have clinical utility in determining preoperative risk, prospective studies that compare postoperative outcomes in frail and non-frail patients and that document both the frailty of patients and the tumor characteristics of meningiomas that were not resected are warranted. Furthermore, additional studies identifying modifiable frailty variables that may benefit from preoperative interventions are needed.

\section{Conclusions}

The present study is the first to examine the relationship between frailty and intracranial meningioma resection. We have demonstrated that increased frailty was an independent risk factor for worse clinical outcomes after meningioma resection, including increased LOS, discharge to a higher level of care, and readmission rates for patients of all ages. These findings were irrespective of patient age, sex, BMI, or tumor size. This finding, once validated prospectively, may provide vital preoperative clinical information to help prognosticate and guide treatment recommendations for older patients considering surgery for intracranial meningioma.

\section{References}

1. Islim AI, Mohan M, Moon RDC, et al. Incidental intracranial meningiomas: a systematic review and meta-analysis of prognostic factors and outcomes. J Neurooncol. 2019;142(2): 211-221.

2. Wiemels J, Wrensch M, Claus EB. Epidemiology and etiology of meningioma. J Neurooncol. 2010;99(3):307-314.

3. Fried LP, Tangen CM, Walston J, et al. Frailty in older adults: evidence for a phenotype. J Gerontol A Biol Sci Med Sci. 2001;56(3):M146-M156.

4. Velanovich V, Antoine H, Swartz A, et al. Accumulating deficits model of frailty and postoperative mortality and morbidity: its application to a national database. J Surg Res. 2013;183(1):104-110.

5. Pazniokas J, Gandhi C, Theriault B, et al. The immense heterogeneity of frailty in neurosurgery: a systematic literature review. Neurosurg Rev. Published online January 17, 2020. doi:10.1007/s10143-020-01241-2

6. Miller EK, Neuman BJ, Jain A, et al. An assessment of frailty as a tool for risk stratification in adult spinal deformity surgery. Neurosurg Focus. 2017;43(6):E3.

7. Leven DM, Lee NJ, Kothari P, et al. Frailty index is a significant predictor of complications and mortality after surgery for adult spinal deformity. Spine (Phila Pa 1976). 2016; 41(23):E1394-E1401.

8. Phan K, Kim JS, Lee NJ, et al. Frailty is associated with morbidity in adults undergoing elective anterior lumbar interbody fusion (ALIF) surgery. Spine J. 2017;17(4):538-544.

9. Yagi M, Fujita N, Okada E, et al. Impact of frailty and comorbidities on surgical outcomes and complications in adult spinal disorders. Spine (Phila Pa 1976). 2018;43(18):1259-1267.

10. Ahmed AK, Goodwin CR, De la Garza-Ramos R, et al. Predicting short-term outcome after surgery for primary spinal tumors based on patient frailty. World Neurosurg. 2017;108: 393-398.

11. Tomlinson SB, Piper K, Kimmell KT, Vates GE. Preoperative frailty score for 30-day morbidity and mortality after cranial neurosurgery. World Neurosurg. 2017;107:959-965.

12. Cloney M, D'Amico R, Lebovic J, et al. Frailty in geriatric glioblastoma patients: a predictor of operative morbidity and outcome. World Neurosurg. 2016;89:362-367.

13. Youngerman BE, Neugut AI, Yang J, et al. The modified frailty index and 30-day adverse events in oncologic neurosurgery. J Neurooncol. 2018;136(1):197-206.

14. de Labra C, Guimaraes-Pinheiro C, Maseda A, et al. Effects of physical exercise interventions in frail older adults: a systematic review of randomized controlled trials. BMC Geriatr. 2015;15(1):154.

\section{Disclosures}

The authors report no conflict of interest concerning the materials or methods used in this study or the findings specified in this paper.

\section{Author Contributions}

Conception and design: Bowers. Acquisition of data: Theriault, Pazniokas, Adkoli, Cho, Rao. Analysis and interpretation of data: Bowers, Theriault, Pazniokas. Drafting the article: Theriault. Reviewed submitted version of manuscript: Schmidt, Cole, Gandhi, Couldwell, Al-Mufti. Approved the final version of the manuscript on behalf of all authors: Bowers. Administrative/ technical/material support: Schmidt. Study supervision: Bowers.

\section{Correspondence}

Christian A. Bowers: University of New Mexico School of Medicine, Albuquerque, NM. cabowers@salud.unm.edu. 